\author{
Lillyan Arely Pérez \\ Universidad Nacional Autónoma de México, México \\ https://orcid.org/0000-0002-3028-5348 \\ Verónica Aideé Ramos \\ Universidad Nacional Autónoma de México, México \\ https://orcid.org/0000-0001-8030-6197 \\ Roland Terborg \\ Universidad Nacional Autónoma de México, México \\ https://orcid.org/0000-0002-7197-5046
}

\title{
Diferentes etapas en el desplazamiento de una lengua indígena: estudio comparativo entre las comunidades de Jesús María, Nayarit y San Juan Juquila Mixes, Oaxaca
}

\begin{abstract}
The attempts to maintain an indigenous language in danger are not always successful. In large part, it is because the causes of language shift are unknown. The main causes of shift must be identified for a successful maintenance of an indigenous language whose gradual shift by Spanish is already in process. The pressures which causes language shift might be identified at the macro level, that is at the national level. However, there are also pressures at the meso and micro levels. These pressures could contribute to make that people stop speaking their native language. Thus, it is not enough to detect only the causes at the macro level to design a language planning that can reverse the process of language shift, but also to consider the regional and local causes. An example are neighboring rural villages with different language vitalities.

There are local factors that can favor either language shift or maintenance. In order to achieve an adequate planning, we have to detect the factors that give rise to different opposing pressures. What can help to identify the factors is the study of language shift in different communities. It is important to detect phenomena that influence in language vitality. In this way we might plan to reverse indigenous language shift. In order to provide the basis for an adequate language planning that promotes the maintenance of indigenous languages in Mexico. This article presents a comparative study of language shift cases. We present a comparison between the Cora community of Jesús María, Nayarit and the Mixe community of San Juan Juquila Mixes, Oaxaca in Mexico. This study is based on the methodological proposal that emerged from the Theory of an Ecology of Pressures and the classification of language shift cases proposed in the same framework. The comparison can help us to identify the causes at the micro level that promote pressures against the maintenance of indigenous languages.

KEYWORDS: Indigenous languages; Language planning; Language shift and maintenance; Ecology of pressures; Utmost common routine.
\end{abstract}

RESUMEN: Los intentos de mantener una lengua indígena en peligro no siempre son exitosos. En gran parte, se debe a que las causas del desplazamiento son desconocidas. Para el mantenimiento exitoso de una lengua 
indígena cuyo desplazamiento paulatino por el español ya está en proceso, en consecuencia, se deben identificar las causas principales del desplazamiento. Las presiones que impulsan hacia el desplazamiento, en gran parte, se pueden identificar en el nivel macro, es decir, a nivel nacional. Sin embargo, también existen presiones en los niveles meso y micro y esas presiones también pueden contribuir a que las personas dejen de hablar su lengua autóctona. Así no basta con detectar solamente las causas a nivel macro para diseñar una planificación del lenguaje que pueda revertir el proceso del desplazamiento, sino también hay que considerar las causas regionales y locales. Como ejemplo nos sirven comunidades rurales, con poca distancia entre ellas, que muestran diferentes panoramas de vitalidad de su lengua. Por lo visto, también hay factores locales que pueden favorecer o el desplazamiento o el mantenimiento de la lengua autóctona.

El problema que enfrentamos, para lograr una buena planificación, es detectar los factores que dan origen a las diferentes presiones opuestas. Lo que puede ayudar a la detección de esos factores es el diagnóstico del avance del proceso del desplazamiento en diferentes localidades. Es importante detectar fenómenos que influyen en el lenguaje, aunque esos mismos fenómenos no sean lingüísticos. De esta manera será posible encontrar una forma de planificar para revertir el desplazamiento de la lengua indígena. En miras de ofrecer las bases para una adecuada planificación del lenguaje que promueva el mantenimiento de las lenguas indígenas en México, así como conocer las causas específicas y factores que influyen en el desplazamiento de las lenguas, este artículo presenta un estudio comparativo de los casos de desplazamiento lingüístico entre la comunidad cora de Jesús María, del estado de Nayarit y la comunidad mixe de San Juan Juquila Mixes, del estado de Oaxaca a partir de la propuesta metodológica que surgió de la Teoría de la Ecología de Presiones y la clasificación de casos de desplazamiento lingüístico propuesta en el mismo marco. La comparación nos podrá ayudar a identificar las causas a nivel micro que promueven las presiones hacia el abandono de la lengua indígena.

Palabras ClaVE: Lenguas indígenas; Planificación del lenguaje; Desplazamiento-mantenimiento de lenguas; Ecología de presiones; Máxima facilidad compartida.

\section{Introducción}

En las últimas décadas el desplazamiento de las lenguas minoritarias se ha acelerado en todo el mundo. Esto que antes no se veía como un problema, actualmente, se percibe cada vez más, como una perdida para la humanidad, así como lo es la extinción de las especies. En la actualidad la organización de las Naciones Unidas para la Educación, la Ciencia y la Cultura (UNESCO 2003) indica que al menos 50\% de las aproximadamente 6 mil lenguas que se hablan en el mundo están en peligro de desaparecer. Hacer frente a la amenaza del desplazamiento de las lenguas minoritarias requiere conocer las causas y los factores que le influyen, así como una planificación del lenguaje en las diferentes áreas donde el fenómeno se presenta. Solo un trabajo conjunto de las comunidades de hablantes, especialistas y académicos, actores sociales e instituciones públicas y privadas puede llegar a mantener las lenguas amenazadas.

Una forma de detectar las causas y los factores particulares que afectan a la situación sociolingüística de una comunidad y poder contribuir al mantenimiento de la diversidad lingüística con acciones adecuadas, es medir el avance del desplazamiento de una lengua en pequeñas comunidades y compararlas tomando en cuenta sus contextos lingüísticos y extralingüísticos.

De esta manera, nuestro objetivo es comparar dos localidades indígenas de México: la comunidad de San Juan Juquila Mixes -Oaxaca- y la comunidad de Jesús María Nayarit-. El interés de este análisis comparativo radica en conocer cómo dos comunidades con características similares presentan etapas de desplazamiento lingüístico distintas. Por una parte, en la comunidad de San Juan Juquila mixes la lengua indígena tiene un grado 
de desplazamiento muy bajo a pesar de que el español va en aumento, por la otra, en la comunidad de Jesús María, la lengua indígena tiene un mayor grado de desplazamiento y el español ya la sobrepasó. Caracterizar cada caso de desplazamiento podrá arrojar luz sobre los factores característicos que le influyen y sus posibles causas, así como comprender mejor la realidad de los hablantes en ambas comunidades.

\section{EI desplazamiento de lenguas}

El fenómeno del desplazamiento se ha convertido en objeto de estudios reflejados en una gran cantidad de publicaciones sobre bilingüismo, política del lenguaje (políticas lingüísticas), lenguas en contacto, ecología lingüística (ecología de lenguas), etc. a nivel mundial (Weinreich 1953; Mackey 1962; Fishman 1991; Edwards 1992; Kaplan \& Baldauf 1997; Spolsky 2004). El desplazamiento o la muerte de lenguas, en estas publicaciones, ha sido tratado como fenómeno secundario o principal, en varios países, en lenguas autóctonas del lugar y en lenguas de migrantes. Presentar un panorama, aunque breve, de los estudios realizados, rebasaría el propósito de este artículo. Un panorama de los avances del siglo pasado se puede encontrar en Sercombe (2002).

También en México abundan los estudios relacionados con el tema que ha despertado el interés por el problema de las lenguas en vías de desaparación. En consecuencia, se publicó en México la Ley General de los Derechos Lingüísticos de los Pueblos Indígenas (2003) cuyo objetivo además de vigilar el reconocimiento y protección de los derechos lingüísticos de las comunidades indígenas, es promover el uso, mantenimiento y desarrollo de las lenguas indígenas en la vida cotidiana.

Dicha ley también requería la fundación del Instituto Nacional de Lenguas Indígenas (INALI) que fue puesto en marcha en 2004. El INALI ha publicado diversos trabajos como el Catálogo de las Lenguas Indígenas Nacionales (2008), alfabetos, libros de texto, material multimedia, literatura en diversas lenguas indígenas, y ha elaborado, además, un Programa de Revitalización, Fortalecimiento y Desarrollo de las Lenguas Indígenas Nacionales (2008).

\section{La teoría de la ecología de presiones, metodología y clasificación}

Nuestra perspectiva de análisis sobre el fenómeno del desplazamiento lingüístico se inspira en los sistemas complejos, debido a que se trata de un fenómeno que envuelve múltiples factores que interactúan dentro de una sociolingüística dinámica (BastardasBoada 2013). Ante esta perspectiva, surge la necesidad de un acercamiento que permita el análisis de esta variedad de factores involucrados en el proceso; en este sentido, teóricamente nos basaremos en la Teoría de la Ecología de Presiones (TEP) y el concepto de Máxima Facilidad Compartida (MFC) desarrollados por Terborg (2006) y Terborg y García Landa (2013). De esta teoría, surge una propuesta metodológica que permite medir el desarrollo del desplazamiento a través de diferentes grupos de edad y la probabilidad de uso de una lengua en cada grupo. De acuerdo con esta metodología se propuso una clasificación preliminar de posibles casos de desplazamiento de una lengua indígena 
PÉREZ; RAMOS; TERBORG - DIFERENTES ETAPAS EN EL DESPLAZAMIENTO... (LI) por la lengua española (LE) (ver Terborg 2016: 26-28). En el marco de esta teoría, encontramos los trabajos de Terborg, Velázquez y Trujillo (2007); Trujillo (2007, 2012); Rico (2010, 2015); Santos (2014); Herrera (2014); Guerra (2016); Sánchez-Fernández, \& Rojas-Berscia (2016), entre otros.

La TEP parte de la concepción de una ecología lingüística como sistema complejo, es decir, una ecología lingüística dinámica, donde todos sus elementos interactúan y están en constante movimiento. La idea central de esta teoría es que los hablantes en situación de contacto lingüístico experimentan diferentes presiones que pueden tener su origen en el interior del sujeto o bien en el contexto exterior. Bajo esta teoría la presión se entiende como "la que un individuo o un grupo siente para actuar de una manera determinada o, en su caso, para evitar algún acto” (Terborg y García-Landa 2011: 36). Así pues, la presión conduce a los hablantes a la acción en general y a la acción lingüística en particular de elegir entre el uso de una lengua u otra. De esta forma, de la TEP deriva una propuesta metodológica para analizar el desplazamiento de una LI por la LE, basada no solo en el sistema lingüístico, sino también en el conocimiento del contexto, los hablantes y sus acciones (Terborg 2016: 18).

Uno de los conceptos centrales de esta teoría es la MFC, en este sentido, se diferencia entre el conocimiento lingüístico individual de cada hablante y el conocimiento lingüístico colectivo, es en este último donde surge la MFC como "la forma más fácil de hablar entre todos" (Terborg 2016: 19), se trata pues, de una suerte de competencia social que implica la elección de una lengua de preferencia y que se mide como mínimo en la interacción de dos hablantes. De esta manera, en contextos de contacto lingüístico, el conocer la MFC permite determinar la presión que experimentan los hablantes hacia el uso de una lengua u otra, la cual, no necesariamente termina siendo su primera lengua, y así de cierta manera, ofrecer un panorama de la situación de desplazamiento o mantenimiento de la lengua que se gesta en una comunidad de hablantes en particular, en un momento determinado. Medir la MFC conlleva varios factores, sin embargo, los esenciales son: El cálculo del conocimiento percibido por los hablantes y el cálculo del uso percibido de la lengua entre diferentes generaciones (Terborg 2016: 22), en este artículo nos concentraremos únicamente en el primero.

La metodología se basa en una muestra significativa de la población mayor a los cinco años de edad y tiene como instrumento un cuestionario sociolingüístico (Terborg y García Landa 2011: 277-279). Este cuestionario consiste en tres secciones, la primera busca recabar conocimientos sociodemográficos de la población; la segunda profundiza sobre el conocimiento tanto de la LI como de la LE, donde los hablantes evalúan el conocimiento que tienen de cada lengua y eligen entre cuatro posibles respuestas: (в) Bien, (Р) Poco, (E) Solo entiende y (N) No entiende; y la tercera que ahonda sobre el uso que hacen los hablantes de ambas lenguas con diferentes generaciones de la población (Terborg 2016: 23). En este sentido, se trata de una evaluación de los hablantes, por lo que el resultado es la percepción que los hablantes tienen sobre el conocimiento y uso que hacen de ambas lenguas en diferentes contextos, esto es a lo que en adelante nos referiremos con la noción de conocimiento percibido.

Con el objeto de observar la situación de desplazamiento lingüístico a través del cálculo de la MFC en los diferentes sectores de la población, se ha realizado una división de la muestra en tres grupos de edad: El grupo A de 5 a 20 años, el grupo B de 21 a 40 
años y el grupo $\mathrm{C}$ de 41 años en adelante. El valor numérico para el conocimiento de cada lengua se calcula en cada grupo, a través de la Fórmula del conocimiento percibido (ver figura 1), esta fórmula se sirve de las respuestas del cuestionario y les asigna un valor que se divide entre el total de hablantes que conforman la muestra, el valor obtenido en cada caso no es menor a cero y ni mayor a 100. La comparación de los valores de conocimiento percibido tanto de la LI como de la LE nos permite obtener la MFC de un grupo de hablantes (Terborg 2016: 20).

$$
\mathrm{CP}=\frac{3 \times \mathrm{B}+2 \times \mathrm{P}+1 \times \mathrm{E}+0 \times \mathrm{N}}{3 \times \mathrm{T}}
$$

Figura 1: Fórmula del Conocimiento Percibido. (Terborg, 2016: 23).

Con base en lo anterior, Terborg (2016: 26-28) ha propuesto una clasificación de casos (ver figura 2) que puede servir como guía sobre la correlación de fenómenos y factores típicos a cada estadio del desplazamiento lingüístico. Esta propuesta es la utilizada para clasificar los casos de desplazamiento de San Juan Juquila Mixes y Jesús María. De esta manera Terborg explica que:

El caso A se refiere a una comunidad donde la Li se encuentra con el valor 100 y la LE con 0 . Aún no hay contacto lingüístico.

El caso в muestra el inicio del contacto con la LE y una ligera variación en los valores de la LI.

El caso C presenta el cambio de la MFC de LI a LE en la comunidad.

El caso D solamente deja la MFC en lengua indígena entre algunas redes sociales, principalmente de las personas mayores (Terborg 2016: 26-28).
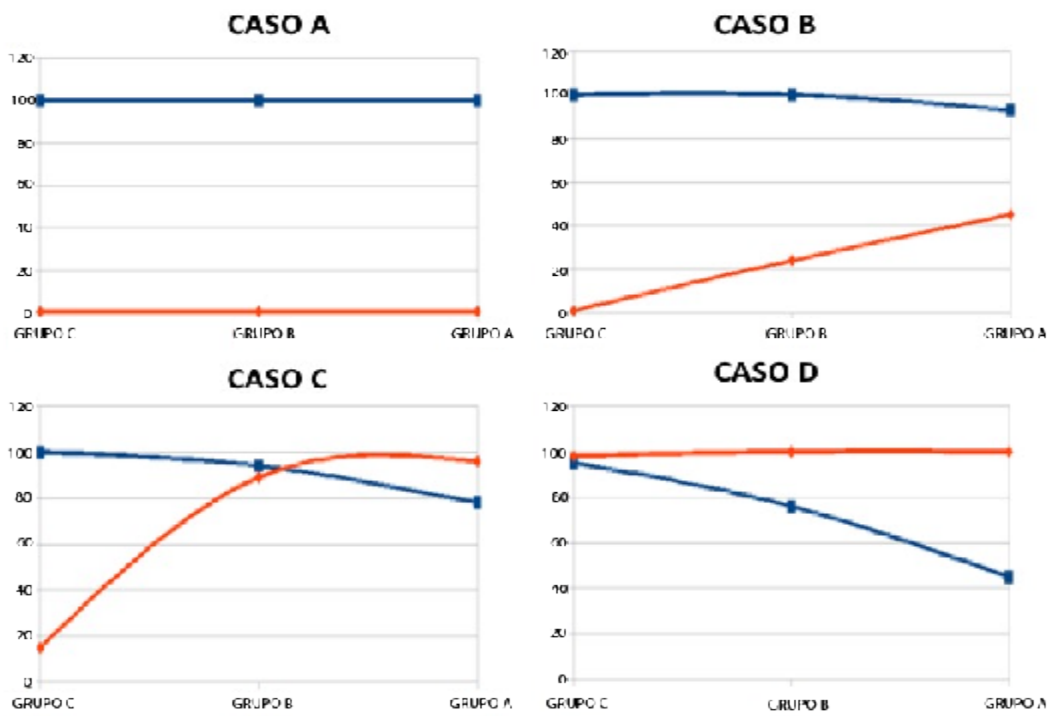

Figura 2: Clasificación de casos de desplazamiento (Terborg 2016: 26-28). 

4. Las comunidades de investigación

El municipio de San Juan Juquila Mixes se ubica a poco más de $117 \mathrm{~km}$ de la Ciudad de Oaxaca en la región de la sierra sur y se considera una población de difícil acceso debido a que parte del camino no está pavimentado y es proclive a deslaves, cuenta además con una población de 1668 habitantes, $52.5 \%$ mujeres, $47.5 \%$ hombres y el $98.79 \%$ de la población se considera indígena (INEG 2010). La lengua que se habla en esta comunidad es el mixe, perteneciente a la familia mixe-zoque en su variante mixe medio del este (INALI 2008: 61-63).

Su principal actividad económica es la agricultura de subsistencia, sin embargo, es un municipio que está considerado con un muy alto grado de marginación debido a que más del $50 \%$ de la población se encuentra en pobreza extrema (SEDESOL s.f.). La precaria situación económica ha generado una fuerte expulsión migratoria hacia la ciudad de Oaxaca, la Ciudad de México y Estados Unidos (CDI 2017).

En lo que respecta a infraestructura, el municipio cuenta únicamente con una unidad médica y 15 escuelas de educación básica y media superior; el grado promedio de escolaridad es de 4.5 años en la población de 15 años o más (CONEval 2010). En cuanto a su forma de gobierno y administración, este municipio cuenta con una organización administrativa de cargos la cual se conforma por un presidente municipal, un síndico y 4 regidores (INAFED s.f.). Su organización social se fundamenta en el cumplimiento de los cargos sociales, el tequio ${ }^{1}$ y las fiestas religiosas que son de gran importancia en la comunidad (CDI 2017).

Una cuestión que destaca al respecto de este territorio, es que históricamente ha sido un territorio en disputa, primero ante sus vecinos zapotecos, después ante los conquistadores españoles, sin embargo son de las pocas comunidades que resistieron el dominio militar. No obstante, existen conflictos en la zona a causa de la ausencia de límites entre comunidades, San Juan Juquila Mixes específicamente, arrastra un conflicto agrario que data de 1975 con la comunidad vecina de San Pedro Ocotepec, desde esa fecha el conflicto ha tenido altibajos, y recientemente en 2017 ha revivido, entre asesinatos, incendios de viviendas y pobladores refugiados (Martínez 2017), estos conflictos han tenido un alto costo en lo político, económico, social y de vidas humanas a lo largo de los años en ambas comunidades.

Por su parte, la comunidad de Jesús María con la que se realizará la comparación es hablante de la variante mariteca de la lengua cora, la cual pertenece a la familia yutoazteca (INALI 2008). Esta comunidad se encuentra ubicada en el municipio El Nayar en la zona noroeste del estado de Nayarit, dentro de los márgenes de la Sierra Madre Occidental, el camino para llegar ahí es una carretera de pavimento, sin embargo, la inestabilidad del terreno complica su acceso, ya que existe un alto riesgo de deslaves y derrumbes rocosos. Al norte comparte su límite territorial con el municipio de Acaponeta y el estado de Durango; al sur con los municipios de Tepic, Santa María del Oro y la Yesca; al oriente lo hace con los estados de Jalisco y Zacatecas, al poniente con los municipios de Santiago Ixcuintla, Acaponeta, Rosamorada y Ruiz (INAFED s.f.). Se estima que la población de

${ }^{1}$ El tequio se considera un trabajo colectivo, obligatorio y gratuito que se presta en beneficio de la comunidad. 
Jesús María es de 2638 habitantes (INEGI 2010), de los cuales el 75\% pertenece al pueblo cora (na'ayeri), un grupo menor al 5\% corresponde al pueblo huichol (wixárika) y el resto se considera de origen mestizo. Históricamente los pueblos de la sierra de "El Gran Nayar" resistieron a diversas incursiones militares de los conquistadores españoles y no fue sino hasta 1722 que el gobierno virreinal logró conquistar esta región (INAFED s.f.).

Desde entonces en esta localidad la religión católica es predominante con un fuerte sincretismo entre esta y los rituales milenarios del pueblo cora, ${ }^{2}$ cuya realización es crucial para su organización social y política, estos ritos basados en un modelo de cargos comunales (Jáuregui 2004) se realizan en vínculo con las autoridades gubernamentales sin la pérdida de la autonomía de las autoridades tradicionales, lo cual se traduce en un símbolo de resistencia. Además de la religión, entre las presiones que experimentan se encuentra la economía del lugar; según datos oficiales el municipio al que pertenece tiene un grado de marginación muy alto, pues el $61.63 \%$ de los pobladores vive en pobreza extrema (SEDSOL s.f.). Según un informe presentado por el Instituto Nacional para la Evaluación de la Educación (2014), la localidad se ha dedicado a actividades relacionadas con la agricultura y la ganadería, sin embargo, la productividad de la tierra ha disminuido considerablemente por la escasez de lluvias, así como la pesca que de acuerdo con algunos pobladores también ha decrecido, lo que incide en la tendencia a migrar a otros lugares como son la costa del estado de Nayarit o los Estados Unidos. La comunidad cuenta con 3 centros de educación inicial, 4 de preescolar, 3 de educación primaria (una escuela rural y dos escuelas de la Dirección General de Educación Indígena), una escuela secundaria técnica y 2 instituciones de nivel medio superior; cuenta con servicios públicos de agua potable y en algunos casos drenaje, una planta de tratamiento de aguas residuales, energía eléctrica, un hospital regional de la Secretaría de Salud, red de telefonía celular (Locreto 2014) y una escasa cobertura de internet. La elección de ambas comunidades para este análisis parte de que se pueden encontrar factores en común entre ellas. Por un lado, ambas son comunidades rurales, relativamente pequeñas y de difícil acceso debido a que están ubicadas entre grandes sistemas montañosos, además ambas son comunidades marginadas con gran afluencia de movimientos migratorios hacia las ciudades cercanas. Por otro lado, las dos comunidades tienen procesos de resistencia histórica a la conquista militar española, aunque no fue así con la conquista espiritual pues en ambas comunidades la religión es católica, si bien con un cierto grado de sincretismo entre los elementos prehispánicos y de la tradición cristiana, esta juega un papel importante dentro de la sociedad. Finalmente, ambas comunidades cuentan en su mayoría con escuelas donde la educación es impartida en español. Sin embargo, con todos estos factores en común, la situación del desplazamiento en cada comunidad es sumamente distinta.

\section{Resultados}

Después de un trabajo de campo en ambas comunidades, donde los datos se recolectaron de la misma forma, los resultados a continuación muestran la situación

\footnotetext{
${ }^{2}$ La ceremonia del mitote, la representación de la judea, la escaramuza de los moros, las pachitas, entre otras. (véase Jáuregui 2004).
} 
PÉREZ; RAMOS; TERBORG - DIFERENTES ETAPAS EN EL DESPLAZAMIENTO... del desplazamiento lingüístico en cada comunidad a través de la comparación entre el conocimiento percibido de la LI y la LE en tres grupos de edad.

En cuanto a la comunidad mixe, de la totalidad de la población de la comunidad se recabaron datos sobre 170 hablantes. Estos datos fueron procesados a través de la Fórmula del conocimiento percibido, con lo que se obtuvo la siguiente información.

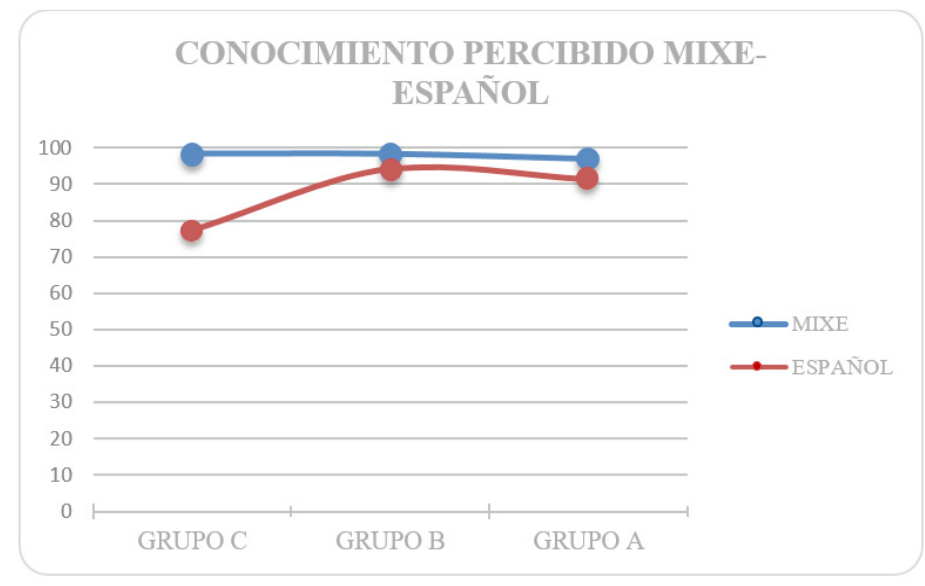

Gráfica 1: Conocimiento percibido Mixe-Español.

Tomando en cuenta que la MFC se revela como el valor más alto entre la Li y la LE, se observa que en cuanto al conocimiento del mixe (Gráfica 1) los valores se mantienen altos en los tres grupos de edad, 98 en los grupos с у в у 96 en el grupo A, mientras que el español comienza con un puntaje de 77 en el grupo C, alcanza un valor de 96 en el grupo в y disminuye a un 91 en el grupo A.

Destaca que en el grupo C el español tiene el valor más bajo de todos los grupos, aunque el mixe no alcanza el valor de 100. El grupo B es el que tiene los valores del mixe y del español más cercanos, por lo que este grupo es altamente bilingüe, y que en el grupo A a pesar de tener los valores del mixe y del español cercanos, ambos se encuentran por debajo de los valores obtenidos por el grupo B.

Es interesante observar que, aunque el conocimiento del mixe ha disminuido del grupo de mayor edad al grupo más joven, este se ha mantenido por arriba del español en los tres grupos de edad, lo que deja ver que la adquisición principal sigue siendo en mixe y por lo tanto la MFC también sigue siendo en mixe. Por otro lado, el conocimiento del español en los tres grupos ha ido en aumento, lo que podría significar un indicio del desplazamiento.

La situación de desplazamiento en la comunidad de San Juan Juquila Mixes se clasifica como un Caso B (ver figura 2), pues muestra el inicio del contacto entre la lengua indígena y el español. En este caso, el mixe se mantiene alto en los grupos C y B, pero comienza a descender en el grupo más joven, mientras que el conocimiento del español comienza bajo en el grupo de mayor edad y se va acercando más al conocimiento de la lengua indígena, conforme el grupo es más joven. 
En lo que respecta a la localidad de Jesús María, para este trabajo se consideraron los datos de 137 hablantes.

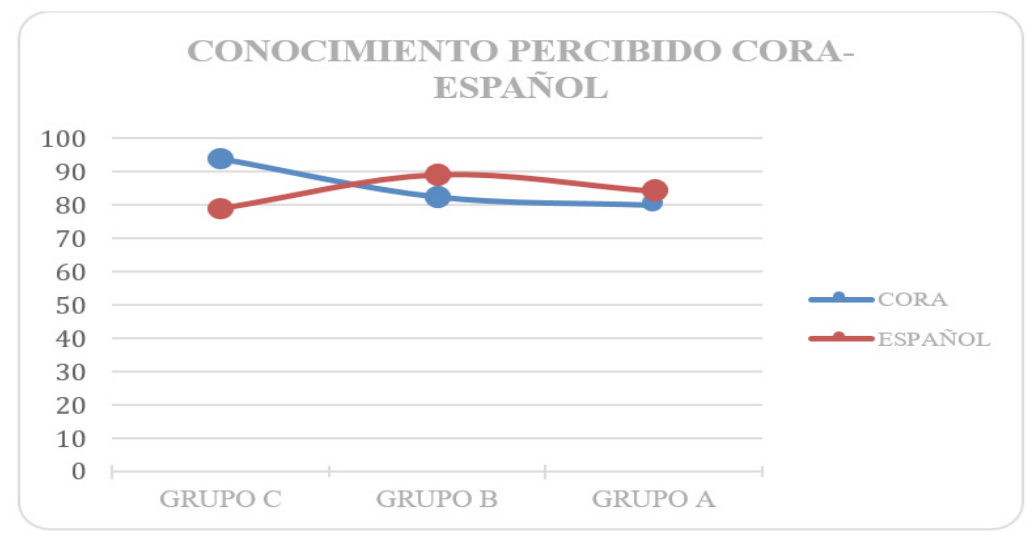

Gráfica 2: Conocimiento percibido Cora-Español.

En este caso podemos observar que el valor del cora se encuentra alto en el grupo C (Gráfica 2) pues alcanza un valor de 93, el valor del cora decae a 82 en el grupo B, y decae otro poco en el grupo A llegando a un valor de 80. Por su parte encontramos que el español comienza con un valor de 78 en el grupo C, sube a 85 en el grupo в у disminuye a 84 en el grupo A.

Es observable que el cora comienza con un valor alto entre los mayores pero disminuye entre los jóvenes y adolescentes, es justamente entre ellos, donde el español se sitúa por encima del cora y se mantiene así en el grupo más jóven. Cabe señalar que aunque en este caso se aprecia un cambio de MFC del cora el español, los valores del cora se mantienen por arriba del 80 en los tres grupos de edad. Por otra parte, el español aunque se sitúa por encima del cora en los grupos в y A, su valor más alto es de 85 , mientras que el valor más alto del cora es de 93 en el grupo c. Finalmente, el hecho de que el español disminuya en el grupo más jovén y el cora mantenga casi el mismo valor que en el grupo B, puede ser una señal de que el cora se habla en el entorno familiar y aún se enseña a los niños.

$\mathrm{Al}$ respecto, debido al cruce que representa el cambio de MFC en la comunidad de Jesús María, la situación de la lengua indígena se clasifica como un caso C (Figura 1) ya que la mayoría de los hablantes de la LI prefieren la LE, incluso en la conversación con otros hablantes de LI, esto probablemente esté relacionado con la presencia de otra LI en la comunidad a causa de los movimientos migratorios del grupo huichol a esta comunidad. Al convivir, cora, huichol y español en esta comunidad, el español se convierte en la lengua en la que esta diversidad coincide y por tanto en la lengua de comunicación más común.

De esta forma, tenemos una comunidad donde la MFC coincide con la LI a pesar del incremento del español y otra comunidad donde la MFC ya cambió y pasó a ser la LE. En el caso de San Juan Juquila Mixes los valores de la Li se mantienen muy altos, por arriba del 95 en los tres grupos de edad y la disminución del valor del grupo de mayor edad al grupo de menor edad es tan solo de dos puntos. Sin embargo, en el caso de Jesús María, el valor 
PÉREZ; RAMOS; TERBORG - DIFERENTES ETAPAS EN EL DESPLAZAMIENTO... más alto del cora es de 93 puntos -en el grupo c- y la disminución del valor entre el grupo de mayor edad y la menor edad es de 13 puntos, por lo que la caída del valor de la LI en este caso es mucho mayor entre los grupos más jóvenes. No obstante, en ambas comunidades aún se mantienen un conocimiento alto de la LI, por lo que es importante implementar estrategias sobre las actitudes de los hablantes hacia su lengua, para evitar que el español desplace completamente a la LI y esta se deje de transmitir.

En cuanto al conocimiento del español, los valores más bajos se encuentran en el grupo C 77 en la comunidad mixe y 78 en la comunidad cora, estos valores aumentan en los grupos в y A, sin embargo en Jesús María se mantienen en 85 y 80 y en Juquila Mixes alcanzan el 96 y el 91 respectivamente, en este sentido, el conocimiento del español es más alto en la comunidad mixe, sin embargo como la Li en dicha comunidad tiene valores aún más altos, no se ha dado un cambio de MFC, si acaso, una tendencia al bilingüismo entre los grupos más jóvenes. En ambas comunidades el grupo más altamente bilingüe es el grupo $\mathrm{B}$, pues expresan tener el mayor conocimiento del español. Ambas comunidades también tienen una pequeña disminución del conocimiento del español en el grupo A que se podría relacionar con que la Li todavía se transmite a los niños en el entorno familiar.

Con el fin de acercarnos a una determinación de las causas específicas del desplazamiento lingüístico en estas comunidades, enfatizaremos a continuación los elementos que tienen en común, pero también aquellos que las distinguen, pues ambas comunidades tienen factores comunes que afectan la situación de desplazamiento de la LI, sin embargo, estos factores se conjugan con características específicas de cada comunidad que influyen la situación de desplazamiento de formas distintas.

Si bien ambas comunidades son aisladas y de difícil acceso debido a su ubicación entre sistemas montañosos, este aislamiento aunado a las condiciones favorables del ecosistema de San Juan Juquila Mixes ha permitido a la población mantener una economía de autoconsumo basada en la agricultura, por lo que pueden mantener su alimentación sin tener que salir constantemente de la comunidad, lo que se ha convertido en una presión favorable para el mantenimiento de la LI en su comunidad. Por su parte, las características del accidentado suelo de Jesús María, han generado parte de la ruptura de ese aislamiento al forzar a los habitantes a salir de la comunidad para conseguir sus productos de consumo, esto ha generado una presión favorable hacia el uso del español, pues fuera de su comunidad esta es la lengua que tienen que utilizar para comunicarse.

Jesús María tienen una población mayor que San Juan Juquila, pero ambas son comunidades relativamente pequeñas, además, ambas tienen procesos históricos de resistencia a la Conquista militar española, sin embargo, la conquista espiritual penetró en ambas comunidades y si bien son comunidades sumamente católicas, sus festividades están llenas de sincretismos porque conservan algunos rituales de sus culturas prehispánicas respectivas.

Por un lado, el territorio Mixe fue sumamente asediado no sólo por los españoles durante la conquista, sino mucho antes por sus vecinos zapotecos y mixtecos, sin embargo, los mixes fueron perseguidos, pero nunca conquistados. Esta historia ha permeado hasta la actualidad en una enemistad que tienen mixes y zapotecos, esto se vuelve importante ya que a pesar de que pueblos zapotecos rodean el territorio mixe y ejercen cierta presión hacia el uso del zapoteco, o bien del español, en Juquila, se ha mantenido el uso del mixe como una especie de código que zapotecas no pueden entender. 
Por otro lado, Jesús María también resistió incursiones militares en la época de la conquista, pues no se da el dominio español hasta 1722, con estas incursiones una serie de movimientos migratorios hacia la comunidad procedentes de los estados colindantes de Jalisco, Zacatecas y Durango, así como de grupos de Huicholes (Jáuregui 2004). Por tal motivo actualmente se pueden encontrar conviviendo en esta comunidad coras, huicholes y mestizos, lo que se convierte en una presión para el desplazamiento del cora, pues lleva a utilizar el español como lengua franca.

Actualmente, la migración es un fenómeno muy presente en ambas comunidades, la marginación y las escasas oportunidades laborales hacen que los habitantes emigren a las ciudades cercanas, cuestión que empuja a los hablantes hacia el uso del español para comunicarse fuera de su comunidad. Por su parte, en Jesús María existe un fenómeno de inmigración a la comunidad de mestizos monolingües del español que apuestan por hacer comercio, lo que también se convierte en una presión hacia el uso del español en esta comunidad.

La organización social basada en el sistema de cargos y el tequio en Juquila sirve de cohesión entre los habitantes de la comunidad y por ende favorece el mantenimiento de la LI, ya que promueve la participación de los habitantes en los asuntos de la comunidad, y los invita a beneficiarse de la ayuda mutua a través del tequio en actividades que van desde el cultivo hasta la construcción. En Jesús María se promueve la cooperación de la población en las fiestas por lo que estas son un fuerte medio de cohesión que refuerza su identidad, lo que a su vez se convierte en una presión favorable para el mantenimiento del cora.

Finalmente, un factor que tienen en común ambas comunidades es la educación en español. En Juquila Mixes todas las instituciones educativas desde preescolar hasta media superior se manejan en español, cuestión que sin duda es una fuerte presión hacia bilingüismo mixe-español en esta comunidad. Por su parte, aunque Jesús María sí cuenta con dos escuelas de educación básica que normativamente se rigen por el sistema de educación indígena, esta no se lleva a cabo debido a que en muchas ocasiones el docente no habla la variante lingüística de esa comunidad, por lo que no hay continuidad de este sistema educativo, y de esta forma también se convierte en una presión que favorece el uso del español.

\section{Consideraciones finales}

La comparación de los casos de desplazamiento de la comunidad cora de Jesús María y la comunidad mixe de San Juan Juquila Mixes nos han permitido conocer cómo dos comunidades con características similares sostienen situaciones de desplazamiento lingüístico distintas, causadas en gran medida por sus rasgos particulares.

Por una parte, en la comunidad de San Juan Juquila mixes encontramos un grado de desplazamiento de la LI muy bajo y un crecimiento en el conocimiento de la LE entre los grupos más jóvenes. Por la otra, en la comunidad de Jesús María, la Li tiene un mayor grado de desplazamiento y la LE ya sobrepasó al cora.

Ambas comunidades comparten el factor del aislamiento geográfico, el cual ha significado una presión favorable hacia el mantenimiento de sus respectivas lenguas, sin 
PÉREZ; RAMOS; TERBORG - DIFERENTES ETAPAS EN EL DESPLAZAMIENTO... embargo, en las dos comunidades la marginación y la consecuente migración a ciudades aledañas, son factores que han causado en cierta medida el desplazamiento de la LI. No obstante, en el caso de San Juan Juquila Mixes, su enemistad con sus vecinos zapotecos, aunada a la cooperación que existe en su organización social dentro de la comunidad, ha reforzado la identidad mixe y por tanto el mantenimiento de su lengua. En cambio, la inmigración de monolingües del español, los matrimonios interculturales entre huicholes y coras, y probablemente otros grupos indígenas, que van vivir a la comunidad de Jesús María, han causado una especie de estratificación social en la población que ha ejercido cierta presión hacia el uso de la LE entre los diversos grupos que habitan esta comunidad.

Por último, podemos señalar que, aunque ambas comunidades tienen situaciones de desplazamiento diferentes y requieren de planificaciones que se adecuen a sus hablantes y que tomen en cuenta las características específicas que las identifican; en ambas el conocimiento de la LI aún es relativamente alto, por lo que parte de las acciones para su mantenimiento, estarían en reforzar las actitudes favorables hacia sus respectivas lenguas indígenas y promover la transmisión intergeneracional. En ese sentido serán necesarios otros estudios para analizar las presiones que determinan el uso de las lenguas en la familia y otros ámbitos, así como las actitudes que expresan los hablantes hacia las lenguas que coexisten en su entorno.

\section{Referencias}

Bastardas-Boada, Albert (2013). Sociolinguistics: Towards a complex ecological view. In Angels Massip-Bonet; Albert Bastardas-Boada (eds.). Complexity perspectives on language, pp. 15-34. Heidelberg: Springer.

Comisión Nacional para el Desarrollo de os Pueblos Indígenas (2017). Etnografía del pueblo mixe de Oaxaca (ayuukjä'äy). Disponible en: https://www.gob.mx/cdi/articulos/etnografia-del-pueblo-mixe-ayuukjaay?idiom $=$ es $>$. Acceso en: 14/09/2017.

Consejo Nacional de Evaluación de la Política de Desarrollo Social (2010). Informe anual sobre la situación de pobreza y rezago social. Disponible en: http://www.microrregiones.gob.mx/catloc/LocdeMun.aspx?tipo= clave\&campo $=$ loc\&ent $=18 \&$ mun $=009$ Acceso en: 23/03/2018.

Consejo Nacional de Evaluación de la Política de Desarrollo Social (2010). Informe anual sobre la situación de pobreza y Rezago Social. Disponible en: https://www.gob.mx/cms/uploads/attachment/file/34534/ Oaxaca 200.pdf Acceso en: 19/03/2018.

Edwards, John (1992). Sociopolitical aspects of language maintenance and loss: Toward a typology of minority language situations. In Willen Fase; Koen Jaspaert; Sjaak Kroon (eds.). Maintenance and loss of minority languages, pp. 37-54. Amsterdam: John Benjamins Publishing Company.

Fishman, Joshua A. (1991). Reversing language shift. Theoretical and empirical foundations of assistance to threatened languages. Clevedon: Multilingual Matters.

Guerra, Roberto (2016). El mantenimiento de una lengua indigena en una comunidad urbana contemporánea de México. El caso del diixhazá o zapoteco en Juchitán de Zaragoza, Oaxaca (Tesis de maestría en lingüística aplicada). Ciudad de México: Universidad Nacional Autónoma de México. 
Herrera, Jesahe (2014). Vitalidad y conservación de lenguas indígenas: el caso del cora en Santa Teresa, Nayarit (Tesis de maestría en lingüística aplicada). Tepic, México: Universidad Autónoma de Nayarit.

Instituto Nacional de Lenguas Indígenas (2008). Catálogo de las lenguas indígenas Nacionales: Variantes Lingüísticas de México con sus autodenominaciones y referencias geoestadísticas. Diario Oficial 31-78. México.

Instituto Nacional de Lenguas Indígenas (2008). Programa de revitalización, fortalecimiento y desarrollo de las lenguas indigenas nacionales. Disponible en: http://www.inali.gob.mx/pdf/PINALI-2008-2012.pdf Acceso en: 17/03/2018.

Instituto Nacional de Estadística y Geografía (2010). Censo de población y vivienda 2010. Población hablante de lengua indígena de 5 años y más. México: Instituto Nacional de Estadística y Geografía. Disponible en: http://internet.contenidos.inegi.org.mx /contenidos/productos/prod_serv/contenidos/espanol/bvinegi/ productos/censos/poblacion/2010/perfil_socio/uem/702825047610_1.pdf Acceso en:19/03/2018.

Instituto Nacional para el Federalismo y el Desarrollo Municipal (sin fecha). Enciclopedia de los Municipios y Delegaciones de México. Disponible en: http://siglo.inafed.gob.mx/enciclopedia/EMM18nayarit/index. html Acceso en: 17/03/2018.

Instituto Nacional para el Federalismo y el Desarrollo Municipal (sin fecha). Enciclopedia de los Municipios y Delegaciones de México. Disponible en: http://www.inafed.gob.mx/work/enciclopedia/EMM20oaxaca/ municipios/20200a.html Acceso en: 19/03/2018

Jáuregui, Jesús (2004). Coras. Pueblos indígenas del México contemporáneo. Comisión Nacional para el Desarrollo de los pueblos Indígenas. Disponible en: http://www.gob.mx/cms/uploads/attachment/ file/11025/coras.pdf Acceso en: 17/03/2018.

Kaplan, Robert; Baldauf, Richard (1997). Language planning: from practice to theory. Clevedon-Philadelphia: Multilingual Matters.

Ley General de los Derechos Lingüísticos de los Pueblos Indígenas (2003). Disponible en: http://www.inali.gob.

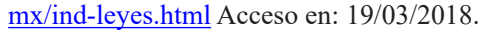

Locreto Genaro (2014). Informe final de la consulta previa, libre e informada en materia de evaluación. Instituto Nacional para la Evaluación de la Educación. Disponible en: http://www.inee.edu.mx/mapacomunidades2016/informes/Jesus.Maria.pdf Acceso en: 17/03/2018.

Mackey, William Francis (1962). The description of bilingualism. Canadian Journal of Linguistics 7(2): 67-73.

Martínez, Ignacio (16 de enero de 2017). Conflicto agrario en San Juan Juquila Mixes, una bomba de tiempo. Rioaxaca. Disponible en: https://www.rioaxaca.com/2017/01/16/conflicto-agrario-en-san-juan-juquilamixes-una-bomba-de-tiempo/ Acceso en: 25/09/2017.

Rico, Gabriel (2010). Mantenimiento y resistencia de la lengua p'urhépecha en Santa Fe de la Laguna, Michoacán (Tesis de maestría en lingüística aplicada). México Distrito Federal: Universidad Nacional Autónoma de México.

Rico, Gabriel (2015). Dinámicas de desplazamiento y resistencia lingüistica en la región p’urhépecha del lago de Pátzcuaro, Michoacán (Tesis de doctorado en lingüística). México Distrito Federal: Universidad Nacional Autónoma de México. 
Sánchez-Fernández, Manuel; Rojas-Berscia, Luis (2016). Vitalidad lingüística de la lengua paipai de Santa Catarina, Baja California. LIAMES-Linguas Indigenas Americanas 16(1): 157-183. doi: https://doi. org/10.20396/liames.v16i1.8646171

Santos, Saul (ed.) (2014). Estudios de vitalidad lingüistica en El Gran Nayar. Tepic: Universidad Autónoma de Nayarit.

Secretaría de Desarrollo Social (sin fecha). Catálogo de localidades. México: SEDEsol. Disponible en: http:// www.microrregiones.gob. $\mathrm{mx} / \mathrm{catloc} /$ LocdeMun. aspx?tipo $=$ clave \&campo $=1$ loc \&ent $=20 \&$ mun $=200$ Acceso en: 19/03/2018.

Sercombe, Peter (2002). Language maintenance and shift: A review of theoretical and regional issues with special reference to Borneo. In Maya Khemlani David (ed.). Methodological and Analytical Issues in Language Maintenance and Language Shift Studies, pp. 1-19. Frankfurt-Meno: Peter Lang.

Spolsky, Bernard (2004). Language policy. Cambridge: Cambridge University Press.

Terborg, Roland (2006). La ecología de presiones en el desplazamiento de las lenguas indígenas por el español. Presentación de un modelo. Forum Qualitative Social Research 7(4): 1-32.

Terborg, Roland (2016). ¿Cómo clasificar el avance del desplazamiento de una lengua indígena para una adecuada planificación del lenguaje? Un primer intento de medición. UniverSOS 13: 11-35.

Terborg, Roland; García-Landa, Laura (eds.) (2011). Muerte y vitalidad de las lenguas indigenas y las presiones sobre sus hablantes. Ciudad de México: CELE-unam.

Terborg, Roland; García-Landa, Laura (2013). The ecology of pressures: Towards a Tool to analize the complex process of language shift and maintenance. In Angels Massip-Bonet; Albert Bastardas-Boada (eds.). Complexity perspectives on language, pp. 219-239. Heidelberg: Springer.

Terborg, Roland; Velázquez, Virna; Trujillo Tames, Alma Isela (2007). La vitalidad de las lenguas indígenas en México: El caso de las lenguas otomí, matlazinca, atzinca y mixe. Martina Schrader-Kniffki; Laura Morgenthaler García (eds.). La Romania en interacción: Entre historia, contacto y política, pp. 611-629. Madrid: Iberoamericana.

Trujillo Tames, Alma Isela (2007). El mantenimiento-desplazamiento de una lengua indígena: El caso de la lengua mixe de Oaxaca, México (Tesis de maestría en lingüística Aplicada). México, Distrito Federal: Universidad Nacional Autónoma de México.

Trujillo Tames, Alma Isela (2012). La vialidad lingüistica de la lengua ayuk o mixe en tres comunidades: Tamazulapam del Espíritu Santo, San Lucas Camotlán y San Juan Guichicovi (Tesis de doctorado en lingüística). México, Distrito Federal: Universidad Nacional Autónoma de México.

United Nations Educational Scientific and Cultural Organization (2003). Vitalidad y peligro de desaparición de las lenguas. Reunión Internacional de Expertos sobre el programa de la UNESCO. Salvaguardia de las lenguas en peligro. Paris.

Weinreich, Uriel (1953). Languages in contact. Nueva York: Linguistic Circle of New York Publication.

Recibido: $15 / 10 / 2018$

Versión revisada y corrigida: 27/11/2018

Aceptado: 4/12/2018. 